This item was submitted to Loughborough's Research Repository by the author.

Items in Figshare are protected by copyright, with all rights reserved, unless otherwise indicated.

\title{
Solid-body rotation and giant circulations in coupled condensates
}

PLEASE CITE THE PUBLISHED VERSION

http://dx.doi.org/10.1088/1361-6455/aa7f83

PUBLISHER

(c) IOP

VERSION

AM (Accepted Manuscript)

LICENCE

CC BY-NC-ND 4.0

REPOSITORY RECORD

Mason, Peter. 2019. "Solid-body Rotation and Giant Circulations in Coupled Condensates". figshare. https://hdl.handle.net/2134/26170. 


\title{
Solid-Body Rotation and Giant Circulations in Coupled Condensates
}

\author{
Peter Mason \\ Department of Physics, Loughborough University, Loughborough, LE11 3TU, United \\ Kingdom \\ E-mail: p.mason@lboro.ac.uk
}

\begin{abstract}
This paper considers a rotating two-component condensate trapped by a harmonic plus Gaussian potential. The ability to tune the parameters to create disk-shaped or annular components, or a combination, means that different rotational regimes can appear in different components. We consider and report on these cases where there is either solid-body rotation in both components, a solid-body rotation in one component and a giant circulation in the other, or a giant circulation in both components.
\end{abstract}

PACS numbers: 03.75.Mn, 05.30.Jp, 67.85.-d

Submitted to: J. Phys. B: At. Mol. Opt. Phys. 


\section{Introduction}

A trapped Bose-Einstein condensate, when it is placed into rotation, will nucleate quantized vortices in the condensate bulk, provided the rotation frequency is above some critical value. As the rotation frequency is increased, so too is the number of vortices [1]. Bretin et al. [2], over a decade previously, experimentally showed this with a series of images of a condensate rotating at different frequencies. Despite the relatively few number of vortices nucleated, it was a beautiful demonstration of the nucleation of, and competition between, quantum vortices.

Often a Bose-Einstein condensate is trapped harmonically, in which case the rotating condensate, provided the rotation frequency is sufficiently large, i.e. there are enough vortices present, will possess a triangular vortex lattice [3, 4]. In such cases, it can be shown that the ground state requires the condensate to be approximately in solidbody rotation. Furthermore, a direct implication of the harmonic trapping potential, is the restriction to a disk-shaped condensate and an upper limit on the rotation frequency $\bar{\Omega}: \bar{\Omega}<\omega$, where $\omega$ is the frequency of the trapping potential.

A number of studies $[2,5]$, primarily with the aim of investigating the "ultrafast" rotation limit, have looked to alleviate this upper limit on the trapping potential, through the addition of a central quartic potential. However, a consequence of imposing a quartic potential to the existing harmonic potential, is to remove the restriction that the shape of the condensate must remain be disk-shaped; we can now have an annularshaped condensate [5]. This allows for the possibility of a rotating condensate in which the geometry is annular, and in which the vortices are nucleated in both the condensate bulk, much as in the case of a purely harmonic trap, or are nucleated inside the annulus, a region where the density is microscopically small, or a combination of both. These latter possibilities gives rise to the appearance of a giant vortex [6] with circulation greater than or equal to one.

The presence of a giant vortex breaks the solid-body rotation of the condensate and instead there is a pure irrotational flow. A number of works have looked to the transition from solid-body rotation to pure irrotational flow. For example, Kavoulakis \& Baym [7] have identified three distinct rotational regimes of interest: (a) a vortex lattice (VL) regime in which the condensate is disk-shaped and possesses many vortices that form a triangular vortex lattice (solid-body rotation), (b) a vortex lattice plus hole (VLH) regime in which the condensate is annular-shaped but there are no (or only a

few) vortices present in the hole (so a giant vortex with a circulation of order 1 ), however the condensate bulk still possesses many vortices that approximately form a triangular lattice, and (c) a giant vortex (GV) regime in which the condensate is annular-shaped and there is a large circulation at the origin (pure irrotational flow). Similar features have been observed in [5], who in addition noted the conditions on the rotation frequency and quartic trapping strength in order to transfer from a disk-shaped condensate to an annular-shaped condensate and [8], who looked to related situations but instead with a harmonic plus Gaussian potential. 
All of the above discussion relates to a single component condensate, however the creation of a condensate mixture leads to a richer physical landscape [9]. The added degrees of freedom naturally present in the system allows for competition between and within the individual components, and between the nucleated topological defects. In this latter case, one can see the presence of triangular vortex lattices, square vortex lattices and vortex sheets [10] (where the vortices from each component form alternating lines).

In this paper we are instead interested in the effects that the condensate mixture has on the geometry of the system and the resulting giant vortex competitions. The creation of a condensate mixture - as, for example in [11, 12, 13] - leads to rotating and non-rotating ground states in which each component may possess a different geometry, and thus the possibility that each component possesses a different type of flow. In order to account for these possibilities, two different trapping potentials can be considered, either a harmonic trapping potential $[14,15,16,17,18,19]$ or an additional term in the potential could be added, such as a quartic [5, 20, 21, 22, 23, 24] or a Gaussian term $[8,25,26,27]$. Here, we will concentrate on the simple harmonic trap as well as the harmonic plus Gaussian trap.

In the case of a condensate mixture with a simple harmonic trapping potential, either both components are disk-shaped or one component is a disk while the other is an annulus $[28,9]$, with a simple increase in the strength of the coupling between the condensates being an adequate control to switch between the two regimes. We can therefore envisage a situation whereby one component is always in the VL regime (disk-shaped and possesses solid-body rotation), while the other component, through an increase in the coupling, transfers from VL to GV. In contrast, in the case of a condensate mixture with a harmonic plus Gaussian trapping potential, all combinations of disk or annular geometry are permitted [14]. This opens up the additional possibility that both components will possess a giant vortex. This paper will investigate in detail these transitions, and competitions between the giant vortices, in mixture condensates.

\section{Energy functional}

The two-component Bose-Einstein condensate at zero temperature in the mean-field regime can be described in terms of two wave functions $\Psi_{1}$ and $\Psi_{2}$ respectively representing component 1 and component 2. The energy functional of the rotating condensate is then given by

$$
\begin{gathered}
E\left[\Psi_{1}, \Psi_{2}\right]=\int \sum_{k=1,2}\left[\frac{\hbar^{2}}{2 m_{k}}\left|\nabla \Psi_{k}\right|^{2}+V_{k}(\boldsymbol{r})\left|\Psi_{k}\right|^{2}-\hbar \bar{\Omega} \Psi_{k}^{*} L_{z} \Psi_{k}+\frac{U_{k}}{2}\left|\Psi_{k}\right|^{4}\right] \\
+U_{12}\left|\Psi_{1}\right|^{2}\left|\Psi_{2}\right|^{2} \mathrm{~d}^{2} r
\end{gathered}
$$

where we have assumed that the characteristic energy of the harmonic oscillator in the $z$ direction is large with respect to the other energy scales so that the geometry is restricted to two dimensions. Here $\boldsymbol{r}=(x, y)$ and the harmonic plus Gaussian magnetic trapping potential is $V_{k}(\boldsymbol{r})=m_{k} \omega_{k}^{2} r^{2} / 2+V_{0} \exp \left(-2 r^{2} / \omega_{0}^{2}\right)$ with trapping frequency $\omega_{k}$ (for $k=1$, 
2 ), energy $V_{0}$ and where $\omega_{0}$ is the waist of the laser (taken here to be centred at the origin). The angular momentum is in the $z$-axis and is defined as $L_{z}=\mathrm{i}[\hat{z} \cdot(\boldsymbol{r} \times \boldsymbol{p})]$, for momentum $\boldsymbol{p}$, and the rotation frequency $\bar{\Omega}=\bar{\Omega} \boldsymbol{e}_{\boldsymbol{z}}$ is taken to apply equally to both components.

The energy functional (1) contains three interaction constants: $U_{k}=$ $\sqrt{8 \pi} \hbar^{2} a_{k} /\left[m_{k} a_{z k}\right] \quad(k=1,2)$ representing the internal interactions in a particular component, and $U_{12}=\sqrt{2 \pi} \hbar^{2} a_{12} /\left[m_{12} \tilde{a}_{z}\right]$ representing the interactions between the two components. The reduced mass is defined as $m_{12}^{-1}=m_{1}^{-1}+m_{2}^{-1}$ and the s-wave scattering lengths are $a_{k}$ and $a_{12}$, with $a_{z k}$ the characteristic length of the harmonic oscillator in the $z$ direction, that is $a_{z k}=\sqrt{\hbar / m_{k} \omega_{z k}}$, with $\omega_{z 1}$ and $\omega_{z 2}$ the frequencies of the confinement in the $z$ direction. The average of the characteristic lengths of the harmonic oscillator in the $z$ direction is defined as $\tilde{a}_{z}=\left(a_{z 1}+a_{z 2}\right) / 2$. To ensure a quasi-two-dimensional condensate, $\hbar \omega_{z k}$ must be large.

To simplify the analysis we set masses and trapping frequencies equal $\left(m_{1}=m_{2} \equiv\right.$ $m$ and $\left.\omega_{1}=\omega_{2} \equiv \omega\right)$ and non-dimensionalise the energy functional according to $\omega^{-1}$, $\hbar \omega$ and $r_{0}=\sqrt{\hbar /\left(2 m_{12} \omega\right)}$ as units of time, energy and length respectively. This results in

$$
E\left[\psi_{1}, \psi_{2}\right]=\int \sum_{k=1,2}\left[\frac{1}{2}\left|\nabla \psi_{k}\right|^{2}+V(r)\left|\psi_{k}\right|^{2}-\Omega \psi_{k}^{*} L_{z} \psi_{k}+\frac{g_{k}}{2}\left|\psi_{k}\right|^{4}\right]+g_{12}\left|\psi_{1}\right|^{2}\left|\psi_{2}\right|^{2} \mathrm{~d}^{2} r
$$

with $V(r)=r^{2} / 2+A \exp \left(-l^{2} r^{2}\right), \Omega=\bar{\Omega} / \omega, A=V_{0} / \hbar \omega$, inverse waist $l=\left(2 \hbar / m \omega \omega_{0}^{2}\right)^{1 / 2}$, $g_{k}=2 U_{k} m_{12} / \hbar^{2}$ and $g_{12}=2 U_{12} m_{12} / \hbar^{2}$. The $\psi_{1}$ and $\psi_{2}$ are the non-dimensionalised wave functions that are subject to the normalisation

$$
\int\left|\psi_{k}\right|^{2} \mathrm{~d}^{2} r=N_{k}
$$

where $N_{k}$ is the total particle number in component $k$. We will assume throughout that there are only ever repulsive interactions, so that $g_{k}, g_{12}$ are always non-negative, and that the intracomponent coupling parameters are in general distinct, making (w.l.o.g) the stipulation that $g_{2} \geq g_{1}$.

\section{A harmonically trapped condensate}

\subsection{Numerical results}

We first begin by considering a purely harmonic trapping potential for which the appropriate energy functional is given by (2) with $A=0$, so that $V(r)=r^{2} / 2$. We keep all parameters constant, except for the intercomponent coupling $g_{12}$ and the rotation $\Omega$. In the following we set $g_{1}=1, g_{2}=2$ and $N_{1}=N_{2}=2000$. These parameters ensure that component 1 is always a disk, whereas component 2 can be either a disk or an annulus, depending on the values we take for $\left(g_{12}, \Omega\right)$. Figure 1 shows example ground state density profiles for both components in either of these scenarios, the first with $\left(g_{12}, \Omega\right)=(0.2,0.6)$, the second with $(1.35,0.6)$. 

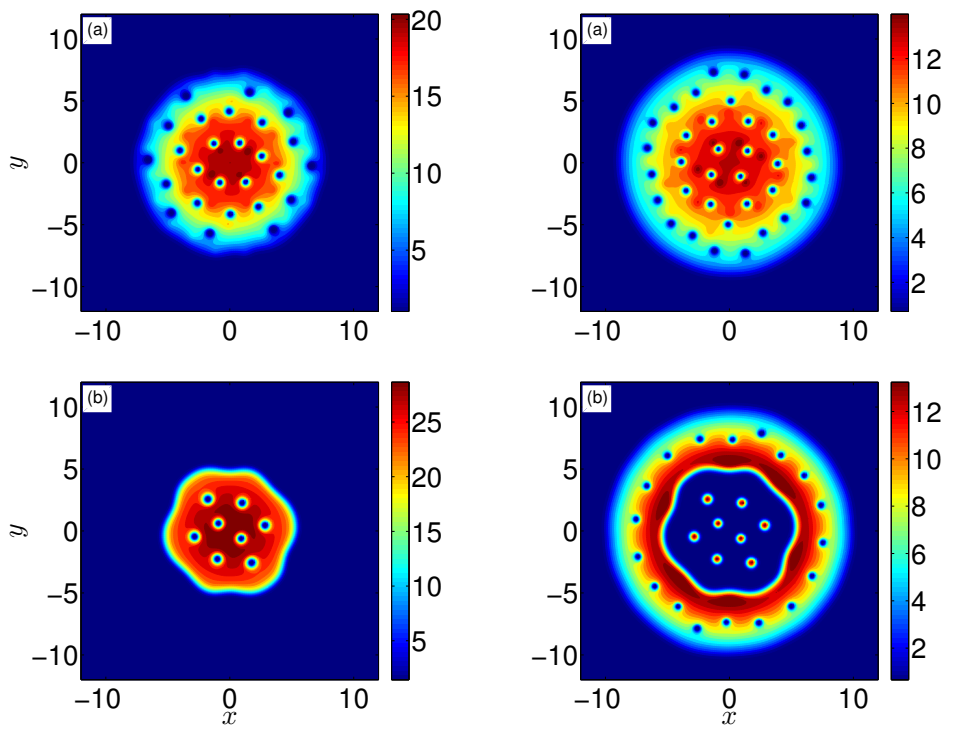

Figure 1. The density profiles of component 1 (left row) and component 2 (right row) for the harmonically trapped condensate with $N_{1}=N_{2}=2000, g_{1}=1, g_{2}=2$, $\Omega=0.6, A=0$ and (a) $g_{12}=0.2$ showing VL+VL and (b) $g_{12}=1.35$ showing $\mathrm{VL}+\mathrm{GV}$.

We see that in Figure 1(a) the two-components are disk-shaped and both contain a triangular vortex lattice (note that these values of intercomponent coupling and rotation impose a triangular, rather than square, lattice $[28,29])$. The condensate thus possesses a solid-body rotation in both components in this regime, which we denote by VL+VL. In Figure 1(b), we see the development of an annulus in component 2, while component 1 remains a disk. Component 1 is therefore still in solid body rotation, whereas component 2 possesses a (small) circulation in the microscopically small density region around the origin. We thus denote this regime by $\mathrm{VL}+\mathrm{GV}$.

\subsection{Thomas-Fermi analysis}

A Thomas-Fermi analysis can be used to find the critical parameters of the system, such as the radii of the components, the chemical potentials and the circulation of the giant vortex. If we use the replacement $\psi_{k}=\sqrt{n_{k}} e^{i \theta_{k}}$ (for $k=1,2$ ), where $n_{k}=\left|\psi_{k}\right|^{2}$ is the density and $\theta_{k}$ is the phase of component $k$, we are able to rewrite the nondimensionalised energy (2) as

$$
\begin{aligned}
E\left[\psi_{1}, \psi_{2}\right]= & \int \sum_{k=1,2}\left\{\frac{1}{2}\left|\nabla \sqrt{n_{k}}\right|^{2}+\left[V(r)+\frac{1}{2} \boldsymbol{v}_{k}^{2}-\boldsymbol{\Omega} \cdot \boldsymbol{r} \times \boldsymbol{v}_{k}\right]\left|\psi_{k}\right|^{2}+\frac{g_{k}}{2}\left|\psi_{k}\right|^{4}\right\} \\
& +g_{12}\left|\psi_{1}\right|^{2}\left|\psi_{2}\right|^{2} \mathrm{~d}^{2} r
\end{aligned}
$$

where we have defined the superfluid velocity in each component as $\boldsymbol{v}_{k}=\nabla \theta_{k}$. We can now invoke the Thomas-Fermi approximation, whereby the kinetic energy is negligible in comparison to the other terms in the energy functional. We thus neglect terms involving 
gradients of density in Eq. (4) $\left[\nabla \sqrt{n_{k}}\right.$ terms $]$. The resulting Thomas-Fermi energy $E_{T F}$ is then defined as

$$
E_{T F}\left[\psi_{1}, \psi_{2}\right]=\int \sum_{k=1,2}\left\{\left[V(r)+\frac{1}{2} \boldsymbol{v}_{k}^{2}-\boldsymbol{\Omega} \cdot \boldsymbol{r} \times \boldsymbol{v}_{k}\right]\left|\psi_{k}\right|^{2}+\frac{g_{k}}{2}\left|\psi_{k}\right|^{4}\right\}+g_{12}\left|\psi_{1}\right|^{2}\left|\psi_{2}\right|^{2} \mathrm{~d}^{2} r .
$$

We lead with the VL+VL state, where both components are in solid-body rotation. Using the fact that the superfluid velocity in component $k$ is equal to the gradient of the phase in that same component, we are thus able to write that $\left(\boldsymbol{v}_{k}^{2} / 2-\boldsymbol{\Omega} \cdot \boldsymbol{r} \times \boldsymbol{v}_{k}\right)=$ $\left(\boldsymbol{v}_{k}-\boldsymbol{v}_{s b}\right)^{2} / 2-\Omega^{2} r^{2} / 2$, where $\boldsymbol{v}_{s b}=\boldsymbol{\Omega} \times \boldsymbol{r}$ is the solid-body velocity, so that the Thomas-Fermi energy reduces to $E_{T F}=E_{s b 1}+E_{s b 2}+E_{I}$ with

$$
\begin{aligned}
E_{s b 1}= & \int \frac{1}{2}\left(\boldsymbol{v}_{1}-\boldsymbol{v}_{s b}\right)^{2}\left|\psi_{1}\right|^{2} \mathrm{~d}^{2} r \\
E_{s b 2}= & \int \frac{1}{2}\left(\boldsymbol{v}_{2}-\boldsymbol{v}_{s b}\right)^{2}\left|\psi_{2}\right|^{2} \mathrm{~d}^{2} r \\
E_{I}= & \int\left(V(r)-\frac{1}{2} \Omega^{2} r^{2}\right)\left(\left|\psi_{1}\right|^{2}+\left|\psi_{2}\right|^{2}\right) \mathrm{d}^{2} r \\
& \quad+\int \frac{g_{1}}{2}\left|\psi_{1}\right|^{4}+\frac{g_{2}}{2}\left|\psi_{2}\right|^{4}+g_{12}\left|\psi_{1}\right|^{2}\left|\psi_{2}\right|^{2} \mathrm{~d}^{2} r .
\end{aligned}
$$

The Thomas-Fermi approximation is valid away from regions where the density curvature becomes significant. In order for the bulk of the condensate to be in the Thomas-Fermi limit, in general, one requires the product of the intracomponent coupling strengths and number of bosons to be large $\left(\tilde{g}_{k}=N_{k} g_{k}\right.$ large). Furthermore, taking a TF approximation on both component wave functions is valid provided $g_{12} \leq \sqrt{g_{1} g_{2}}$ (see [9] for more precise requirements). As a consequence our analysis does not cover the region $\sqrt{g_{1} g_{2}}<g_{12}$.

In the first regime $(\mathrm{VL}+\mathrm{VL})$ we have solid-body rotation in both components. In analogy to single component condensates [5] we then set $\boldsymbol{v}_{1}=\boldsymbol{v}_{2}=\boldsymbol{v}_{s b}$ so that $E_{s b 1}=0$ and $E_{s b 2}=0$ and the energy reduces simply to $E_{T F}=E_{I}$. At this level of approximation, we have from [9], the expressions for the radii and chemical potentials. Both components are disk-shaped, and we can write down the radii of component 1 and component 2, respectively, as

$$
\begin{aligned}
& R_{1}=\left[\frac{4}{\left(1-\Omega^{2}\right)}\left(\frac{N_{1} g_{1} \Gamma_{12}}{\pi \Gamma_{2}}\right)\right]^{1 / 4}, \\
& R_{2}=\left[\frac{4}{\left(1-\Omega^{2}\right)}\left(\frac{N_{2} g_{2}}{\pi}+\frac{N_{1} g_{12}}{\pi}\right)\right]^{1 / 4},
\end{aligned}
$$

and the respective chemical potentials as

$$
\begin{aligned}
& \mu_{1}=\Gamma_{2}\left[\left(1-\Omega^{2}\right)\left(\frac{N_{1} g_{1} \Gamma_{12}}{\pi \Gamma_{2}}\right)\right]^{1 / 2}+\frac{g_{12}}{g_{2}}\left[\left(1-\Omega^{2}\right)\left(\frac{N_{2} g_{2}}{\pi}+\frac{N_{1} g_{12}}{\pi}\right)\right]^{1 / 2}(8 \mathrm{a},) \\
& \mu_{2}=\left[\left(1-\Omega^{2}\right)\left(\frac{N_{2} g_{2}}{\pi}+\frac{N_{1} g_{12}}{\pi}\right)\right]^{1 / 2} .
\end{aligned}
$$


Mason \& Aftalion [9] gave an explicit expression for the value of $g_{12}$ at which the transition of component 2 from a disk to being an annulus occurs (the miscibility/immiscibility transition). The expression is

$$
g_{12}^{c}=\frac{N_{1} g_{1}}{2\left(N_{1}+N_{2}\right)}+\frac{1}{2}\left[\left(\frac{N_{1} g_{1}}{N_{1}+N_{2}}\right)^{2}+\frac{4 N_{2} g_{1} g_{2}}{N_{1}+N_{2}}\right]^{1 / 2} .
$$

Using the above chosen parameters $\left(N_{1}=N_{2}=2000, g_{1}=1, g_{2}=2\right)$ we find $g_{12}^{c}=1.28$, which is in agreement with our numerical simulations which give $g_{12}^{c} \approx 1.27$. Note that this expression is independent of $\Omega$. However one must be careful when comparing these two values: when $g_{1}<g_{12}<g_{12}^{c}$ the effective potential felt by component 2 at the origin is negative so that the density for component 2 in the domain $r<R_{1}$ is convex. In particular, when $g_{12} \rightarrow g_{12}^{c}$, the density of component 2 at and near the origin is small. Thus, the rotating condensate is predisposed to favour the nucleation of (possibly more than) one vortex in component 2 near the origin, and so, since the analysis to Eq. (9) does not take into account the appearance of vortices, the rotation to some extent, disguises the true value of $g_{12}$ at which the annulus develops.

In the $\mathrm{VL}+\mathrm{GV}$ state component 2 is annular and there is a giant vortex present at the centre. A number of papers have looked at the giant vortex [5, 7, 8, 30] and we follow their introduction of a quanta of circulation. Taking component 2 to have a large circulation at the centre is equivalent to it possessing pure irrotational flow, for which the velocity is just $\nu_{2} / r$. Here $\nu_{2}$ is the quantum of circulation, and we have given it the subscript ' 2 ' to associate it with component 2 . Therefore we let $\boldsymbol{v}_{2}=\nu_{2} / r$. Minimisation of the free energy per particle, $F=E-\mu_{2} N_{2}$, with respect to $\nu_{2}$ yields

$$
\Omega N_{2}=2 \pi \nu_{2} \int \frac{\left|\psi_{2}\right|^{2}}{r} \mathrm{~d} r
$$

In the Thomas-Fermi limit we still have $\left(\boldsymbol{v}_{1}^{2} / 2-\boldsymbol{\Omega} \cdot \boldsymbol{r} \times \boldsymbol{v}_{1}\right)=\left(\boldsymbol{v}_{1}-\boldsymbol{v}_{s b}\right)^{2} / 2-\Omega^{2} r^{2} / 2$ so that under the assumption that component 1 is in solid-body rotation, $E_{s b 1}=0$, and the energy reduces to $E_{T F}=E_{p f 2}+\bar{E}_{I}$ with

$$
\begin{aligned}
E_{p f 2}= & \int\left(\frac{\nu_{2}^{2}}{2 r^{2}}-\Omega \nu_{2}\right)\left|\psi_{2}\right|^{2} \mathrm{~d}^{2} r \\
\bar{E}_{I}= & \int\left(V(r)-\frac{1}{2} \Omega^{2} r^{2}\right)\left|\psi_{1}\right|^{2}+V(r)\left|\psi_{2}\right|^{2} \mathrm{~d}^{2} r \\
& +\int\left(\frac{g_{1}}{2}\left|\psi_{1}\right|^{4}+\frac{g_{2}}{2}\left|\psi_{2}\right|^{4}\right)+g_{12}\left|\psi_{1}\right|^{2}\left|\psi_{2}\right|^{2} \mathrm{~d}^{2} r .
\end{aligned}
$$

From this, the general profiles of the components are simply

$$
\begin{aligned}
& \left|\psi_{1}\right|^{2}=\frac{1}{g_{1} \Gamma_{12}}\left[\mu_{1}-\frac{g_{12}}{g_{2}} \tilde{\mu}_{2}-\Gamma_{2} V(r)+\frac{1}{2} \Omega^{2} r^{2}+\frac{1}{2 r^{2}} \frac{g_{12}}{g_{2}} \nu_{2}^{2}\right], \\
& \left|\psi_{2}\right|^{2}=\frac{1}{g_{2} \Gamma_{12}}\left[\tilde{\mu}_{2}-\frac{g_{12}}{g_{1}} \mu_{1}-\Gamma_{1} V(r)-\frac{1}{2} \frac{g_{12}}{g_{1}} \Omega^{2} r^{2}-\frac{1}{2 r^{2}} \nu_{2}^{2}\right],
\end{aligned}
$$


if both components co-exist and

$$
\begin{array}{ll}
\left|\psi_{1}\right|^{2}=\frac{1}{g_{1}}\left[\mu_{1}-V(r)+\Omega^{2} r^{2} / 2\right], & \left|\psi_{2}\right|^{2}=0, \\
\left|\psi_{2}\right|^{2}=\frac{1}{g_{2}}\left[\tilde{\mu}_{2}-V(r)-\nu_{2}^{2} /\left[2 r^{2}\right]\right], & \left|\psi_{1}\right|^{2}=0,
\end{array}
$$

otherwise. Here, $\tilde{\mu}_{2}=\mu_{2}+\Omega \nu_{2}$ and $\Gamma_{k}=1-g_{12} / g_{k}$. Thus it remains to find the radii, chemical potentials and the quanta of circulation for given parameters $g_{1}, g_{2}, g_{12}, N_{1}$, $N_{2}$, and $\Omega$. This can be achieved by taking the normalisation conditions from (3) and the expression that results from the minimisation of the free energy (10) for density profiles described in (12) and (13).

We define the boundaries of the annular component 2 to be at $r=R_{2}^{-}$and $r=R_{2}^{+}$, with $R_{2}^{-}<R_{1}<R_{2}^{+}$. By evaluating the density in (12) at each of the boundaries $r=R_{2}^{-}$and $r=R_{1}$ and (13b) at $r=R_{2}^{+}$, we get the following expressions involving the chemical potentials and the quanta of circulation:

$$
\begin{aligned}
\tilde{\mu}_{2} & =\frac{1}{2} R_{2}^{+^{2}}+\frac{\nu_{2}^{2}}{2 R_{2}^{+^{2}}}, \\
\mu_{1}-\frac{g_{12}}{g_{2}} \tilde{\mu}_{2} & =\frac{1}{2} R_{1}^{2}\left(\Gamma_{2}-\Omega^{2}\right)-\frac{g_{12}}{g_{2}} \frac{\nu_{2}^{2}}{2 R_{1}^{2}}, \\
\tilde{\mu}_{2}-\frac{g_{12}}{g_{1}} \mu_{1} & =\frac{1}{2} R_{2}^{-2}\left(\Gamma_{1}+\frac{g_{12}}{g_{1}} \Omega^{2}\right)+\frac{v_{2}^{2}}{2 R_{2}^{-2}} .
\end{aligned}
$$

Completion of the integrals (3) and (10), and using (14), results in the following expressions

$$
\begin{aligned}
& \frac{4 N_{1} g_{1} \Gamma_{12}}{\pi}=\left(\Gamma_{2}-\Omega^{2}\right) R_{1}^{4}+\frac{g_{12}}{g_{2}}\left(\Gamma_{1}+\frac{g_{12}}{g_{1}} \Omega^{2}\right) R_{2}^{-4} \\
& +4 \frac{g_{12}}{g_{2}} \nu_{2}^{2} \log \left(\frac{R_{1}}{R_{2}^{-}}\right) \\
& \frac{4 N_{2} g_{2} \Gamma_{12}}{\pi}=-\frac{g_{12}}{g_{1}}\left(\Gamma_{2}-\Omega^{2}\right) R_{1}^{4}+\Gamma_{12} R_{2}^{+^{4}}-\left(\Gamma_{1}+\frac{g_{12}}{g_{1}} \Omega^{2}\right) R_{2}^{-4} \\
& -4 \nu_{2}^{2}\left[\log \left(\frac{R_{1}}{R_{2}^{-}}\right)+\Gamma_{12} \log \left(\frac{R_{2}^{+}}{R_{1}}\right)\right] \\
& \frac{N_{2} g_{2} \Omega \Gamma_{12}}{\pi \nu_{2}}=\left(\Gamma_{1}+\frac{g_{12}}{g_{1}} \Omega^{2}\right)\left[\log \left(\frac{R_{1}}{R_{2}^{-}}\right)+1\right] R_{2}^{-2}+\frac{g_{12}}{g_{1}}\left(\Gamma_{2}-\Omega^{2}\right) R_{1}^{2} \\
& +\Gamma_{12}\left[\log \left(\frac{R_{2}^{+}}{R_{1}}\right)-1\right] R_{2}^{+^{2}} \\
& +\nu_{2}^{2}\left[\frac{\Gamma_{12}}{R_{2}^{+^{2}}} \log \left(\frac{R_{2}^{+}}{R_{1}}\right)+\frac{1}{R_{2}^{-2}} \log \left(\frac{R_{1}}{R_{2}^{-}}\right)\right] \text {, }
\end{aligned}
$$

and

$$
\nu_{2}^{2}\left(\frac{\Gamma_{12}}{R_{2}^{+^{2}}}-\frac{1}{R_{2}^{-2}}+\frac{g_{12}^{2}}{g_{1} g_{2} R_{1}^{2}}\right)=\frac{g_{12}}{g_{1}}\left(\Gamma_{2}-\Omega^{2}\right) R_{1}^{2}+\left(\Gamma_{1}+\frac{g_{12}}{g_{1}} \Omega^{2}\right) R_{2}^{-2}-\Gamma_{12} R_{2}^{+^{2}} .
$$




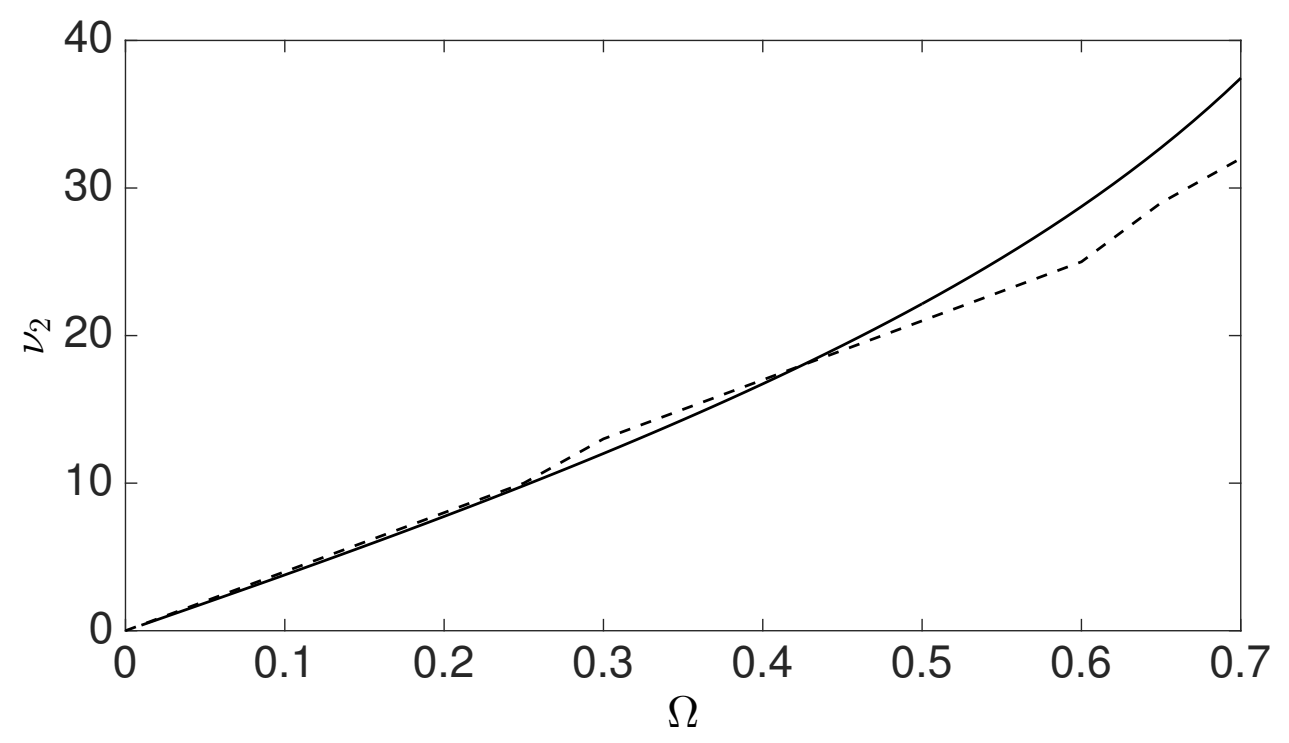

Figure 2. The quanta of circulation $\nu_{2}$ as a function of the rotation $\Omega$ calculated through a Thomas-Fermi analysis (15) (dashed lines) and numerically (solid lines) for the harmonically trapped condensate with $N_{1}=N_{2}=2000, g_{1}=1, g_{2}=2, g_{12}=1.35$ and $A=0$.

We numerically solve these equations for the parameter set $\left(g_{1}, g_{2}, N_{1}, N_{2}, A\right)=$ $(1,2,2000,2000,0)$. In Figure 2 we plot the resulting values for $\nu_{2}$ as a function of $\Omega$ with a fixed $g_{12}=1.35$, and compare it to those values calculated from a full numerical simulation on the coupled GP equations. One could also plot $\nu_{2}$ as a function of $g_{12}$ for fixed $\Omega$, however we find that $\nu_{2}$ is approximately constant over a wide range of values of $g_{12}$.

\section{A harmonic plus Gaussian trap}

In the case of a rotating two-component condensate with a simple harmonic trapping, as considered in Section 3, the only (symmetry preserving) geometry permitted by the system is that where both components are disks or where one component is a disk with the other an annulus surrounding the disk. A choice of a harmonic plus Gaussian trapping potential, $V(r)=r^{2} / 2+A \exp \left(-l^{2} r^{2}\right)$, allows us to study both the above configurations but with the additional configuration in which both components are annuli. This opens up the possibility that we can have giant circulations in both components. Specifically we are interested in the relative magnitudes of the giant circulations, something that we can investigate through an analytical Thomas-Fermi analysis backed up by numerical simulations.

In this section we concentrate on the GV+GV regime in the Thomas-Fermi limit. In this regime, both components have pure irrotational flow, and so as before, we now introduce $\boldsymbol{v}_{1}=\nu_{1} / r$ to represent the persistent flow at the centre of component 1 . 
Solid-Body Rotation and Giant Circulations in Coupled Condensates

Minimisation of the free energy per particle with respect to $\nu_{1}$ yields

$$
\Omega N_{1}=2 \pi \nu_{1} \int \frac{\left|\psi_{1}\right|^{2}}{r} d r .
$$

The energy thus reduces to $E_{T F}=E_{p f 1}+E_{p f 2}+\tilde{E}_{I}$ with

$$
\begin{aligned}
E_{p f 1}= & \int\left(\frac{\nu_{1}^{2}}{2 r^{2}}-\Omega \nu_{1}\right)\left|\psi_{1}\right|^{2} \quad d^{2} r \\
\tilde{E}_{I}= & \int V(r)\left(\left|\psi_{1}\right|^{2}+\left|\psi_{2}\right|^{2}\right) \quad d^{2} r \\
& +\int\left(\frac{g_{1}}{2}\left|\psi_{1}\right|^{4}+\frac{g_{2}}{2}\left|\psi_{2}\right|^{4}\right)+g_{12}\left|\psi_{1}\right|^{2}\left|\psi_{2}\right|^{2} \quad d^{2} r
\end{aligned}
$$

and with $E_{p f 2}$ defined as previously. We set $R_{2}^{-}<R_{1}^{-}<R_{1}^{+}<R_{2}^{+}$and so the general profiles of the components are simply

$$
\begin{aligned}
& \left|\psi_{1}\right|^{2}=\frac{1}{g_{1} \Gamma_{12}}\left[\tilde{\mu}_{1}-\frac{g_{12}}{g_{2}} \tilde{\mu}_{2}-\Gamma_{2} V(r)-\frac{1}{2 r^{2}}\left(\nu_{1}^{2}-\frac{g_{12}}{g_{2}} \nu_{2}^{2}\right)\right], \\
& \left|\psi_{2}\right|^{2}=\frac{1}{g_{2} \Gamma_{12}}\left[\tilde{\mu}_{2}-\frac{g_{12}}{g_{1}} \tilde{\mu}_{1}-\Gamma_{1} V(r)-\frac{1}{2 r^{2}}\left(\nu_{2}^{2}-\frac{g_{12}}{g_{1}} \nu_{1}^{2}\right)\right],
\end{aligned}
$$

if both components co-exist and

$$
\left|\psi_{2}\right|^{2}=\frac{1}{g_{2}}\left[\tilde{\mu}_{2}-V(r)-\frac{1}{2 r^{2}} \nu_{2}^{2}\right], \quad\left|\psi_{1}\right|^{2}=0
$$

if only component 2 exists. Here, $\tilde{\mu}_{1}=\mu_{1}+\Omega \nu_{1}$.

By evaluating the density in (18) at each of the boundaries of the annular component $1\left(r=R_{1}^{-}, r=R_{1}^{+}\right)$, noting that $\left|\psi_{1}\right|^{2}=0$ at $r=R_{1}^{+}$and $r=R_{1}^{-}$and equating, we get the following expressions involving the chemical potentials and the quanta of circulation:

$$
\begin{aligned}
& \tilde{\mu}_{1}-\frac{g_{12}}{g_{2}} \tilde{\mu}_{2}=\frac{1}{2} R_{1}^{+^{2}} \Gamma_{2}+A \Gamma_{2} \exp \left(-l^{2} R_{1}^{+^{2}}\right)+\frac{1}{2 R_{1}^{+^{2}}}\left(\nu_{1}^{2}-\frac{g_{12}}{g_{2}} \nu_{2}^{2}\right), \\
& \tilde{\mu}_{1}-\frac{g_{12}}{g_{2}} \tilde{\mu}_{2}=\frac{1}{2} R_{1}^{-^{2}} \Gamma_{2}+A \Gamma_{2} \exp \left(-l^{2} R_{1}^{-2}\right)+\frac{1}{2 R_{1}^{-2}}\left(\nu_{1}^{2}-\frac{g_{12}}{g_{2}} \nu_{2}^{2}\right),
\end{aligned}
$$

and repeating the procedure for component 2 we get

$$
\begin{aligned}
& \tilde{\mu}_{2}=\frac{1}{2} R_{2}^{+^{2}}+A \exp \left(-l^{2} R_{2}^{+^{2}}\right)+\frac{1}{2{R_{2}^{+2}}_{2}^{2}}, \\
& \tilde{\mu}_{2}=\frac{1}{2} R_{2}^{-^{2}}+A \exp \left(-l^{2} R_{2}^{-2}\right)+\frac{1}{2 R_{2}^{-2}} \nu_{2}^{2} .
\end{aligned}
$$


Solid-Body Rotation and Giant Circulations in Coupled Condensates

Equating (20a)-(20b) and (21a)-(21b) separately then gives

$$
\begin{aligned}
& \nu_{1}^{2}=\Gamma_{2} R_{1}^{-^{2}} R_{1}^{+^{2}}+\frac{g_{12}}{g_{2}} R_{2}^{-^{2}} R_{2}^{+^{2}} \\
&+2 A\left\{\frac{\Gamma_{2} R_{1}^{-^{2}} R_{1}^{+^{2}}}{X_{1}}\left[\exp \left(-l^{2} R_{1}^{+^{2}}\right)-\exp \left(-l^{2} R_{1}^{-2}\right)\right]\right. \\
&+\frac{g_{12}}{g_{2}} \frac{R_{2}^{-^{2}} R_{2}^{+^{2}}}{X_{2}}\left[\exp \left(-l^{2}{R_{2}^{+2}}^{2}-\exp \left(-l^{2} R_{2}^{-^{2}}\right)\right]\right\}, \\
& \nu_{2}^{2}=R_{2}^{-2} R_{2}^{+^{2}}+2 A \frac{R_{2}^{-^{2}} R_{2}^{+^{2}}}{X_{2}}\left[\exp \left(-l^{2} R_{2}^{+^{2}}\right)-\exp \left(-l^{2} R_{2}^{-2}\right)\right],
\end{aligned}
$$

where we have defined $X_{k}={R_{k}^{+2}}^{2} R_{k}^{-2}$ for $k=1,2$. Completion of the integrals (3), (10) and (16), and using (20)-(21), results in the following expressions

$$
\begin{aligned}
\frac{N_{1} g_{1} \Gamma_{12}}{\pi \Gamma_{2}}= & \frac{1}{4} X_{1}\left(R_{1}^{+^{2}}+R_{1}^{-^{2}}\right)-\frac{1}{2}{R_{1}^{+}}^{2} R_{1}^{-2} \ln \left(\frac{R_{1}^{+^{2}}}{R_{1}^{-2}}\right)+A X_{1} \exp \left(-l^{2} R_{1}^{+^{2}}\right) \\
& +A \exp \left(-l^{2} R_{1}^{-2}\right)\left[\exp \left(-l^{2} X_{1}\right)-1\right]\left[R_{1}^{-^{2}}+\frac{1}{l^{2}}-\frac{{R_{1}^{+}}^{2} R_{1}^{-2}}{X_{1}} \ln \left(\frac{R_{1}^{+^{2}}}{R_{1}^{-2}}\right)\right],
\end{aligned}
$$

and

$$
\begin{aligned}
\frac{g_{1} N_{1} \Omega \Gamma_{12}}{\pi \nu_{1}}= & {\left[\frac{1}{2} \Gamma_{2}\left(R_{1}^{+^{2}}+R_{1}^{-2}\right)+A \Gamma_{2} \exp \left(-l^{2} R_{1}^{+^{2}}\right)\right.} \\
& \left.+\frac{A \Gamma_{2} R_{1}^{-^{2}}}{X_{1}} \exp \left(-l^{2} R_{1}^{-2}\right)\left(\exp \left(-l^{2} X_{1}\right)-1\right)\right] \ln \left(\frac{R_{1}^{+^{2}}}{R_{1}^{-2}}\right) \\
& -\Gamma_{2} X_{1}-A \Gamma_{2} \exp \left(-l^{2} R_{1}^{-2}\right)\left[\exp \left(-l^{2} X_{1}\right)-1\right]-2 A \Gamma_{2} \int_{R_{1}^{-}}^{R_{1}^{+}} \frac{1}{r} \exp \left(-l^{2} r^{2}\right) \mathrm{d} r
\end{aligned}
$$

and

$$
\begin{aligned}
& \frac{N_{1} g_{12}}{\pi}+\frac{N_{2} g_{2}}{\pi}=\frac{1}{4} X_{2}\left(R_{2}^{-^{2}}+R_{2}^{+^{2}}\right)-\frac{1}{2}{R_{2}^{-2}}^{+_{2}^{2}} \ln \left(\frac{R_{2}^{+^{2}}}{{R_{2}^{-2}}^{2}}\right)+A X_{2} \exp \left(-l^{2} X_{2}\right) \exp \left(-l^{2} R_{2}^{-^{2}}\right) \\
& +A \exp \left(-l^{2} R_{2}^{-2}\right)\left[\exp \left(-l^{2} X_{2}\right)-1\right]\left[{R_{2}^{-2}}^{-2} \frac{1}{l^{2}}-\frac{{R_{2}^{+}}^{2} R_{2}^{-2}}{X_{2}} \ln \left(\frac{R_{2}^{+^{2}}}{R_{2}^{-2}}\right)\right],
\end{aligned}
$$


and

$$
\begin{aligned}
\frac{g_{12} N_{1} \Omega}{\pi \nu_{1}}+\frac{g_{2} N_{2} \Omega}{\pi \nu_{2}} & =\left[\frac{1}{2}\left(R_{2}^{-^{2}}+R_{2}^{+^{2}}\right)+A \exp \left(-l^{2} R_{2}^{+^{2}}\right)\right. \\
& \left.+\frac{A R_{2}^{-^{2}}}{X_{2}} \exp \left(-l^{2} R_{2}^{-2}\right)\left[\exp \left(-l^{2} X_{2}\right)-1\right]\right] \ln \left(\frac{R_{2}^{+^{2}}}{R_{2}^{-2}}\right) \\
& -X_{2}-A \exp \left(-l^{2} R_{2}^{-2}\right)\left[\exp \left(-l^{2} X_{2}\right)-1\right]-2 A \int_{R_{2}^{-}}^{R_{2}^{+}} \frac{1}{r} \exp \left(-l^{2} r^{2}\right) \mathrm{d} r .
\end{aligned}
$$

The above equations provide us with information on the values of $\nu_{1}$ and $\nu_{2}$. To proceed, we first perform numerical simulations in the specific case where populations densities and interaction strengths are equal, i.e. we set $N_{1}=N_{2}=2000$ and $g_{1}=g_{2}=1$, together with $A=70, l=0.3$ and $g_{12}=0.8$. These numerical simulations are compared to the exact solutions of Eq.'s (22)-(23) in Fig. 3(a). One can clearly see the analytics and numerics are in good agreement for small values of the rotation (up to $\Omega=0.25$ ), however once this value is passed, agreement is lost. The explanation for this is straightforward: for $\Omega \leq 0.25$ no vortices are nucleated in the condensate bulk (of either component) and hence the analytical estimates above, that rely on the assumption of pure irrotation flow, are valid. Conversely, when $\Omega \sim 0.3$, vortices are nucleated in the bulk - inducing a solid body rotation - that is not accounted for in the estimates. Further (second and third) rows of vortices are nucleated when $\Omega \sim 0.4$ and $\Omega \sim 0.5$. A plot of the density of component 1 is shown in Fig. 4 for the case of $\Omega=0.1$, 0.3 and 0.5 .

Therefore, to properly test our estimates we wish to avoid significant vortex nucleation in the bulk, and furthermore to distinguish between the quanta of circulation. To this end, a more general set of parameters is chosen: $N_{1}=N_{2}=500, g_{1}=1, g_{2}=2$, $A=100, l=0.3$ and $g_{12}=0.8$. The results of these simulations are shown in Fig. 3(b) and again are compared to the exact solutions of Eq.s (22)-(23). Here we see that agreement holds for a wider range of values of $\Omega$. It is noted that vortices are nucleated in the bulk condensate when $\Omega \sim 0.6$.

\section{Conclusion}

This paper has considered rotation of a two-component Bose-Einstein condensate in cases where the trapping potential is either harmonic or harmonic plus Gaussian. Subject to the choice of parameters and trapping potential, different geometries can be realised, and we here concentrated on those that created an annulus in either or both of the components. Together with the rotation, this allows for the creation of a giant vortex, and the possibility to transition from solid-body rotation to a persistent flow as the parameters are changed. The governing equations in the Thomas-Fermi limit for

each case are written down and numerically solved; these results are then compared with 

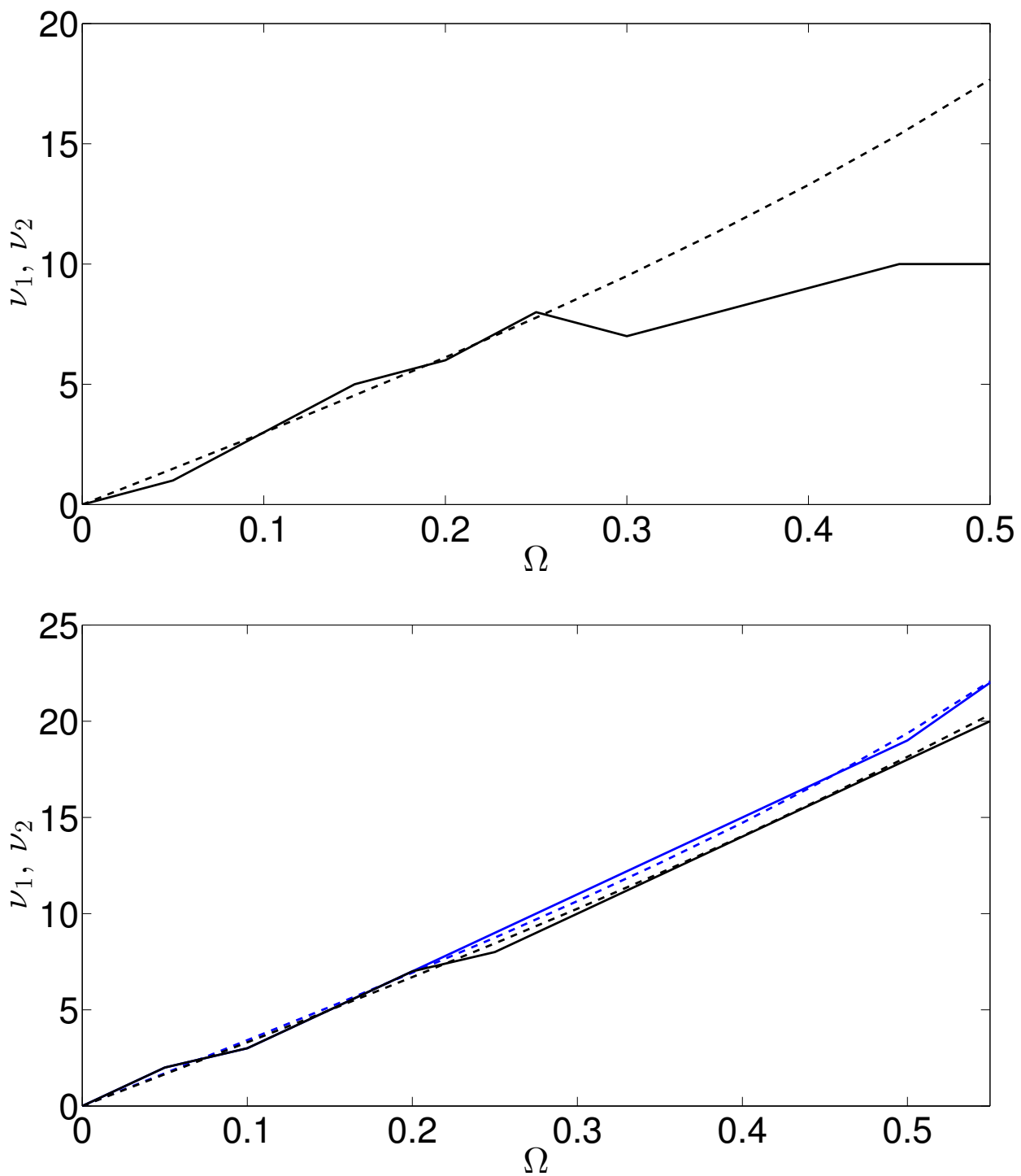

Figure 3. The quanta of circulation $\nu_{1}$ (black lines) and $\nu_{2}$ (blue lines) as a function of the rotation calculated numerically (solid lines) and by solving exactly Eq.'s (22)-(23) (dashed lines) for the condensate with a harmonic plus Gaussian trapping potential with parameters: (a) $N_{1}=N_{2}=2000, g_{1}=g_{2}=1, g_{12}=0.8, A=70$ and $l=0.3$, and (b) $N_{1}=N_{2}=500, g_{1}=1, g_{2}=2, g_{12}=0.8, A=100$ and $l=0.3$.

a full numerical simulation of the GP equations. Through the numerical simulations we are able to determine the competition between the giant vortices, and to establish three distinct regimes of interest: a vortex lattice-vortex lattice regime, a vortex-lattice and giant vortex regime, and a giant vortex-giant vortex regime. 

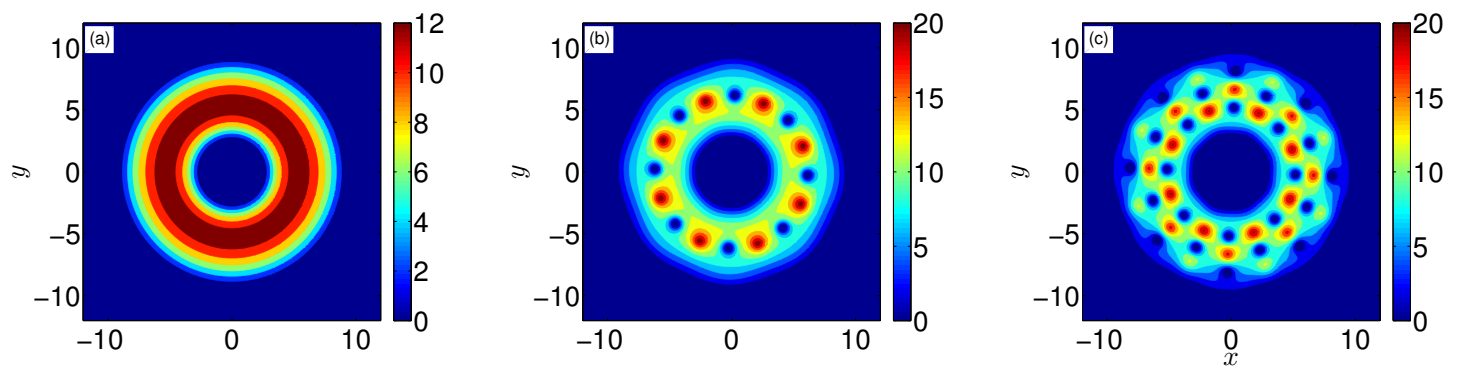

Figure 4. The density profiles of component 1 for the condensate with a harmonic plus Gaussian trapping potential with parameters $N_{1}=N_{2}=2000, g_{1}=g_{2}=1$, $g_{12}=0.8, A=70$ and $l=0.3$ at different rotations: (a) $\Omega=0.1$, (b) $\Omega=0.3$ and (c) $\Omega=0.5$.

\section{Acknowledgements}

The author wishes to thank Amandine Aftalion for discussions that took place throughout this work.

\section{References}

[1] C. J. Pethick \& H. Smith, Bose-Einstein Condensation in Dilute Gases, Cambridge University Press (2002).

[2] V. Bretin, S. Stock, Y. Seurin, and J. Dalibard, Phys. Rev. Lett., 92, 050403 (2004).

[3] A. L. Fetter, Rev. Mod. Phys., 81, 647 (2009).

[4] N. R. Cooper, Advances in Physics, 57, 539-616 (2008).

[5] A. L. Fetter, B. Jackson and S. Stringari, Phys. Rev. A, 71, 013605 (2005).

[6] T. P. Simula, A. A. Penckwitt, and R. J. Ballagh, Phys. Rev. Lett., 92, 060401 (2004).

[7] G. M. Kavoulakis \& G. Baym, New Journal of Physics, 5, 1367-2630 (2003).

[8] A. Aftalion and P. Mason, Physical Review A, 81, 023607 (2010).

[9] P. Mason and A. Aftalion, Phys. Rev. A, 84, 033611 (2011).

[10] K. Kasamatsu and M. Tsubota, Phys. Rev. A, 79, 023606 (2009).

[11] G.Modugno, M. Modugno, F. Riboli, G. Roati, and M. Inguscio, Phys. Rev. Lett., 89, 190404 (2002).

[12] G. Thalhammer, G. Barontini, L. de Sarlo, J. Catani, F.Minardi, and M. Inguscio, Phys. Rev. Lett. 100, 210402 (2008).

[13] G. Ferrari, M. Inguscio, W. Jastrzebski, G. Modugno, G. Roati, and A. Simoni, Phys. Rev. Lett., 89, $053202(2002)$.

[14] P. Mason, Eur. Phys. J. B, 86, 453 (2013).

[15] P. Kuopanportti, N. V. Orlova, and M. V. Milosevic, Phys. Rev. A, 91, 043605 (2015).

[16] J. Jin, S. Zhang, W. Han and Z. Wei, J. Phys. B: At. Mol. Opt. Phys., 46, 075302 (2013).

[17] S. Yang, Q. Wu, S. Zhang, and S. Feng, Phys. Rev. A, 77, 033621 (2008).

[18] K. Kasamatsu, M. Tsubota and M. Ueda, Phys. Rev. A, 71, 043611 (2005).

[19] J Christensson et al., New Journal of Physics, 10, 033029 (2008).

[20] C.-H. Hsueh, T.-L. Horng, S.-C. Gou, and W. C. Wu, Phys. Rev. A, 84, 023610 (2011).

[21] E. Ö. Karabulut, F. Malet, G. M. Kavoulakis, and S. M. Reimann, Phys. Rev. A, 87, 043609 (2013).

[22] Y. Liu and S.-Y. Zhang, Chin. Phys. B, 25, 090304 (2016).

[23] H. Fu \& E. Zaremba, Phys. Rev. A, 73, 013614 (2006). 
[24] J.-K. Kim \& A. L. Fetter, Phys. Rev. A, 72, 023619 (2005).

[25] O. Morizot et al., Phys. Rev. A, 74, 023617 (2006).

[26] C. Ryu et al., Phys. Rev. Lett., 99, 260401 (2007).

[27] L.H. Wen and X.B. Luo, Laser Phys. Lett., 9, 618624 (2012).

[28] K. Kasamatsu, M. Tsubota and M. Ueda, Int. J. Modern Phy. B, 19, 1835-1904 (2005).

[29] A. Aftalion, P. Mason and J. Wei, Phys. Rev. A, 85, 033614 (2012).

[30] U. R. Fischer \& G. Baym, Phys. Rev. Lett., 90, 140402 (2003). 